\title{
COMMENTARY
}

\section{Histone Deacetylase Inhibitory Approaches for the Management of Osteoarthritis}

\author{
Chandra K. Singh, ${ }^{*}$ Minakshi Nihal, ${ }^{*}$ and Nihal Ahmad ${ }^{* \dagger}$
}

From the Department of Dermatology, ${ }^{*}$ University of Wisconsin, Madison; and the William S. Middleton VA Medical Center, ${ }^{\dagger}$ Madison, Wisconsin

Histone acetylation, a dynamic cellular process, is critical in regulating gene transcription in a variety of ways. This process is tightly regulated by the antagonistic actions of two unique families of enzymes, the histone deacetylases (HDACs) and histone acetyltransferases. HDACs remove acetyl groups $(\mathrm{O}=\mathrm{C}-\mathrm{CH} 3)$ from histones to induce the generation of a compressed, transcriptionally repressed chromatin structure. The HDAC proteins are classified into four groups based on DNA sequence similarity and functional activities. ${ }^{1}$ Classic HDAC proteins (class I, II, and IV) possess a zinc-dependent active site and are further grouped into 11 subtypes named chronologically HDAC 1 to 11 . HDAC class III proteins require the $\mathrm{NAD}^{+}$cofactor for activity and are known as sirtuins, a family of seven known members (SIRT 1 to 7).

The HDAC inhibitors (HDACi) are currently being extensively investigated for the management of a variety of diseases, including inflammatory conditions and cancer. In this issue of The American Journal of Pathology, Makki and Haqqi ${ }^{2}$ have found that the drug vorinostat, which is a class I and II HDAC inhibitor, blocks IL-1 $\beta$-induced expression of matrix metallopeptidase (MMP)-13, tumor necrosis factor- $\alpha$, and other catabolic factors in human osteoarthritis $(\mathrm{OA})$ chondrocytes obtained from the human knee cartilage. ${ }^{2}$ Moreover, vorinostat was also found to rescue the collagen type II $\alpha$ and aggrecan proteoglycan expression in OA chondrocytes, which were down-regulated by IL-1 $\beta$. In addition, the authors have demonstrated that IL6-stimulated MMP-13 expression was independent of IL-1 $\beta$ stimulation and was blocked by vorinostat, suggesting that vorinostat inhibits IL-6 signaling in chondrocytes. In this commentary, we discuss the implications of these findings and the potential of HDAC inhibition in the management of OA.

\section{Role of HDACs in OA Pathogenesis}

$\mathrm{OA}$ is a degenerative disease of the joint resulting from the breakdown of joint cartilage and underlying bone. OA is a highly prevalent form of arthritis of the knee and hip, affecting approximately $3.8 \%$ of the human population, globally as of $2010 .^{3}$ OA is alleged to be triggered by mechanical stress on the joint leading to low-grade inflammation and progressive loss of the cartilage matrix. ${ }^{4} \mathrm{OA}$ starts with high cell proliferation and increased synthesis of matrix proteins, cytokines, and other inflammatory mediators by chondrocytes, which are the only cell type found in the cartilage matrix. ${ }^{4}$ Chondrocytes possess low metabolic activity, little regenerative capacity, but may survive in the absence of a vascular supply and relatively under hypoxic conditions. These cells are eventually accountable for remodeling and keeping the structural and functional integrity of the cartilage matrix. ${ }^{4}$ Therefore, chondrocytes have been extensively used to understand the pathogenesis of OA.

HDACs are modulated in several pathological conditions, including cancer. However, only a limited number of studies have implicated HDACs in the pathogenesis of OA. HDAC1 and HDAC2 protein levels are elevated in primary human chondrocytes, which is consistent with repression of cartilage-specific genes. ${ }^{5}$ HDAC4 is known to be a major regulator of chondrocyte hypertrophy throughout skeletogenesis. The abnormal expression of HDAC4 in osteoarthritic cartilage suggests its involvement in promoting the catabolic activity of chondrocytes associated with $\mathrm{OA}$ pathogenesis. ${ }^{6}$ A significant elevation of HDAC7 in the cartilage of OA patients is reported to contribute to cartilage degradation via promoting MMP-13. ${ }^{7}$ These studies suggest

Supported in part by NIH grants R01AR059130 and R01CA176748 and the Department of Veterans Affairs VA Merit Review award 1I01BX001008 (N.A.).

Accepted for publication August 5, 2016.

Disclosures: None declared.

Address correspondence to Nihal Ahmad, Ph.D., Department of Dermatology, University of Wisconsin, 1300 University Ave, Medical Sciences Center, Room 423, Madison, WI 53706. E-mail: nahmad@wisc.edu 
that inhibiting certain HDACs may be useful in the management of OA. However, future studies are required to unravel the roles of specific HDACs in OA pathophysiology because the use of specific HDACi may be more effective.

Regarding the role of class III HDACs in OA, most studies have focused on the sirtuin SIRT1. SIRT1 activity is essential for cartilage-specific extracellular components aggrecan proteoglycan and collagen type II $\alpha$ in human chondrocytes derived from OA patients. ${ }^{8}$ Many studies have assessed the biological actions of SIRT1 in cartilage homeostasis and OA. Gabay et $\mathrm{al}^{9}$ assessed the in vivo relevance of SIRT1 in cartilage biology and demonstrated that SIRT1 heterozygous haploinsufficient $\left(\mathrm{SIRT1}^{+-}{ }^{-}\right.$) mice are growth delayed with early symptoms of mild OA by middle age, whereas $\mathrm{SIRT}^{-l-}$ pups exhibit skeletal malformations and die before weaning age. Overexpression of SIRT1 in chondrocytes by expression plasmid under stimulation with IL- $1 \beta$ reduces the expression of catabolic enzymes, such as a disintegrin and metalloproteinase with thrombospondin motifs (ADAMTS)5 and matrix metalloproteinases (MMPs 1, 2, 9, 10, 11, 12, and 13), as well as acetylation of NF- $\kappa \mathrm{B} / \mathrm{p} 65$, suggesting the protective role of SIRT $1 .{ }^{10}$ Recently, Li et al ${ }^{11}$ showed that the intra-articular injection of the grape antioxidant resveratrol, a known SIRT1 activator, prevents the destruction of OA cartilage by increasing SIRT1 and inhibiting hypoxiainducing factor- $2 \alpha$ expression in mouse OA cartilage and in IL-1 $\beta$-treated human chondrocytes. Together, these studies suggest that SIRT1 activators may be useful in the management of OA, especially in a preventive setting.

\section{Potential Use of HDAC Inhibitors in OA Management}

Current therapeutic options for the management of OA either have short-term efficacy or are ineffective and fail to reverse/stop pathophysiological events involved in disease progression. Progressions of catabolic and anabolic mediators have been shown to play crucial roles in articular cartilage homeostasis and in the development and progression of OA. These inflammatory mediators include tumor necrosis factor- $\alpha$, interleukins (IL-1 $\beta$, IL-6, IL-8, IL-15, IL-17, IL-21), prostaglandin E2, and fibroblast growth factor 2; signaling mediators such as NF- $\mathrm{BB}$, mitogen-activated protein kinase, protein kinase $\mathrm{C}-\delta$, and $\beta$-catenin; and proteases such as MMP-1, MMP-3, MMP-9, MMP-13, and a disintegrin and metalloproteinase with thrombospondin motifs (ADAMTS-4 and ADAMTS-5). ${ }^{12}$ Interestingly, $\mathrm{IL}-1 \beta$ is an inducer of MMPs and ADAMTS proteases and suppresses the cartilage matrix formation. Recent studies also indicate that MMP-13 is overexpressed in the late stage of OA, and its knockdown in mice resists OA cartilage damage. Makki and Haqqi ${ }^{2}$ have found that vorinostat blocks IL-1 $\beta$-induced expression of MMP-13 in human OA chondrocytes. As the first report suggesting HDAC inhibitor vorinostat as a suppressor of IL-6-induced signaling events in $\mathrm{OA}$, this study has a potential of opening new avenues in OA management. ${ }^{2}$
Indeed, hyperacetylation of histone proteins up-regulates cell cycle inhibitors $\left(\mathrm{p} 21^{\mathrm{Cip} 1}, \mathrm{p} 27^{\mathrm{Kip} 1}, \mathrm{p} 16^{\mathrm{INK} 4}\right)$, represses inflammatory cytokines (IL-1, IL- 8 , tumor necrosis factor- $\alpha$, TGF- $\beta$ ), and down-regulates immune stimulators (IL-6, IL-10, CD154). ${ }^{13}$ Furthermore, aberrant HDAC activity has been linked to a wide variety of pathological conditions. Thus, inhibiting HDAC activity offers potential solutions to prevent or reverse these conditions. HDACi includes a range of naturally occurring as well as synthetic compounds, which differ in terms of function and HDAC specificity. Some HDACi (eg, trichostatin A, vorinostat) are panHDAC inhibitors, which inhibit the activity of class I and II HDACs, whereas others are class/isoform-selective inhibitors (eg, FK-228 inhibits HDAC 1 and 2). HDACi have been in use in psychiatry and neurology as mood stabilizers and anti-epileptics for some time. Recently, HDACi have emerged as a possible treatment for cancers and inflammatory diseases. ${ }^{14}$

As of now there are 609 HDACi-related human clinical trials completed/ongoing (https://clinicaltrials.gov; last accessed July 21, 2016); however, none of them are related to OA. One study has assessed the safety and efficacy of an oral HDACi givinostat (ITF2357) in systemic-onset juvenile idiopathic arthritis (12 weeks at a dosage of $1.5 \mathrm{mg} / \mathrm{kg}$ per day to 17 patients). This study has found a significant therapeutic benefit of ITF2357, specifically with regard to the arthritic component of the disease, and showed an excellent safety profile. ${ }^{15}$

Vorinostat has emerged as a popular and promising HDACi that is orally bioavailable and acts as a broad spectrum inhibitor of class I and II HDACs (HDAC 1 to 10). Vorinostat, chemically known as suberoylanilide hydroxamic acid, and clinically as Zolinza, is clinically the most advanced HDACi that was discovered through extensive evaluations of small polar molecules proficient in inhibiting HDAC enzymes. ${ }^{16}$ Approximately half of all of the reported clinical trials on HDACi are with vorinostat. Vorinostat was first approved in 2006 by the US Food and Drug Administration for the treatment of cutaneous manifestations in patients with cutaneous T-cell lymphoma in whom other treatments have failed. The study by Makki and Haqqi, ${ }^{2}$ showing vorinostat-mediated suppression of MMP-13 through inhibition of IL- 6 in chondrocytes, suggests the potential of vorinostat as a therapeutic agent for the management of OA.

The use of HDACi in OA management is also supported by some other studies as well. Chabane et $\mathrm{al}^{17}$ have shown that HDACi trichostatin A (TSA) and butyric acid suppress IL-1 $\beta$-induced inflammatory mediators nitric oxide and prostaglandin E2 production, inducible nitric oxide synthase and cyclooxygenase- 2 expression, as well as proteoglycan degradation in human chondrocytes. Chen et al ${ }^{18}$ have shown alleviation of OA by TSA in an experimental model of OA induced in New Zealand rabbits through unilateral anterior cruciate ligament transection on left knee joints. The authors found that elevated levels of MMP-1, MMP-3, MMP-13 and 
IL-1 were significantly reduced by TSA. TSA has also been shown to suppress both IL-1-dependent and IL1-independent MMP-13 mRNA expression in human knee chondrocytes. ${ }^{7}$ HDACi TSA suppresses the mechanical stressinduced expression of RUNX-2 and ADAMTS- 5 by inhibiting the activation of mitogen-activated protein kinase signaling [extracellular signal-regulated kinase, c-Jun NH2-terminal kinase (JNK), p38 mitogen-activated protein kinase (p38)] in human chondrocytes. ${ }^{19}$ Vorinostat down-regulates inducible nitric oxide synthase, MMP-1, and MMP-13 expression and up-regulates tissue inhibitor of metalloproteinases-1 expression in human chondrocytes. Furthermore, vorinostat selectively represses IL-1 $\beta$-induced p38 and extracellular signal regulated kinase $1 / 2$ activation without affecting JNK activation and also inhibits NF- $\kappa$ B signaling. ${ }^{20}$ Culley et $\mathrm{al}^{21}$ have shown the protective effect of TSA on cartilage degeneration in a destabilization of the medial meniscus mouse model of OA. These studies indicate that the inhibition of HDACs could have a beneficial effect in the management of osteoarthritic conditions.

\section{Naturally 0ccurring HDAC Inhibitors}

Many of the HDACi are derived naturally from microbial metabolites (eg, TSA, isolated from Streptomyces hygroscopicus). Allyl derivatives from garlic were the first molecules identified to affect histone acetylation status. Other prominent plant-based HDACi includes sulforaphane (found in cruciferous vegetables) and quercetin (found in a variety of fruits and beverage). ${ }^{1}$ Because HDACi seems to possess therapeutic potential against a variety of diseases, there is increasing interest in the potential of dietary compounds that possess HDAC inhibitory activities. However, some of the natural agents appear to have dual effects on HDACs. For example, resveratrol is a reported activator of class III HDAC SIRT1 but also has been shown to inhibit the other 11 human HDACs (class I, II, and IV). ${ }^{22}$ Other examples are of curcumin and genistein, which exhibit both HDAC inhibitory and histone acetylation activator activities. ${ }^{1}$ It appears that further research is needed to determine whether the naturally occurring HDAC modulators may be useful in OA management.

\section{Conclusions}

HDACs post-translationally alter histone and nonhistone proteins, which affect the final gene expression outcome. Clearly, there is an increasing interest in the therapeutic use of HDACi in the treatment of a variety of disorders linked to HDAC dysfunction. Certain HDACs have been shown to be deregulated in OA. However, this area of research is still in its infancy and detailed investigations are needed to understand the functional and therapeutic significance of specific HDACs in OA. Determining which HDAC is to be targeted and whether a selective HDACi or a pan-HDACi would be useful in the therapeutic management of OA is an important area of future research. The article by Makki and Haqqi ${ }^{2}$ in this issue, is an important effort in this direction that provides substantial evidence for the potential use of vorinostat in OA management.

\section{References}

1. Bassett SA, Barnett MP: The role of dietary histone deacetylases (HDACs) inhibitors in health and disease. Nutrients 2014, 6:4273-4301

2. Makki MS, Haqqi TM: Histone deacetylase inhibitor vorinostat (SAHA) suppresses interleukin-1 $\beta$ induced matrix metallopeptidase-13 expression by inhibiting interleukin-6 in osteoarthritis chondrocyte. Am J Pathol 2016, 186:2701-2708

3. March L, Smith EU, Hoy DG, Cross MJ, Sanchez-Riera L, Blyth F, Buchbinder R, Vos T, Woolf AD: Burden of disability due to musculoskeletal (MSK) disorders. Best Pract Res Clin Rheumatol 2014, 28: 353-366

4. Goldring MB, Goldring SR: Osteoarthritis. J Cell Physiol 2007, 213: 626-634

5. Hong S, Derfoul A, Pereira-Mouries L, Hall DJ: A novel domain in histone deacetylase 1 and 2 mediates repression of cartilage-specific genes in human chondrocytes. FASEB J 2009, 23:3539-3552

6. Lu J, Sun Y, Ge Q, Teng H, Jiang Q: Histone deacetylase 4 alters cartilage homeostasis in human osteoarthritis. BMC Musculoskelet Disord 2014, 15:438

7. Higashiyama R, Miyaki S, Yamashita S, Yoshitaka T, Lindman G, Ito Y, Sasho T, Takahashi K, Lotz M, Asahara H: Correlation between MMP-13 and HDAC7 expression in human knee osteoarthritis. Mod Rheumatol 2010, 20:11-17

8. Dvir-Ginzberg M, Gagarina V, Lee EJ, Hall DJ: Regulation of cartilage-specific gene expression in human chondrocytes by SirT1 and nicotinamide phosphoribosyltransferase. J Biol Chem 2008, 283: $36300-36310$

9. Gabay O, Oppenhiemer H, Meir H, Zaal K, Sanchez C, Dvir-Ginzberg M: Increased apoptotic chondrocytes in articular cartilage from adult heterozygous SirT1 mice. Ann Rheum Dis 2012, 71:613-616

10. Matsushita T, Sasaki H, Takayama K, Ishida K, Matsumoto T, Kubo S, Matsuzaki T, Nishida K, Kurosaka M, Kuroda R: The overexpression of SIRT1 inhibited osteoarthritic gene expression changes induced by interleukin-1beta in human chondrocytes. J Orthop Res 2013, 31: $531-537$

11. Li W, Cai L, Zhang Y, Cui L, Shen G: Intra-articular resveratrol injection prevents osteoarthritis progression in a mouse model by activating SIRT1 and thereby silencing HIF-2alpha. J Orthop Res 2015, 33:1061-1070

12. Lee AS, Ellman MB, Yan D, Kroin JS, Cole BJ, van Wijnen AJ, Im HJ: A current review of molecular mechanisms regarding osteoarthritis and pain. Gene 2013, 527:440-447

13. Lin HS, Hu CY, Chan HY, Liew YY, Huang HP, Lepescheux L, Bastianelli E, Baron R, Rawadi G, Clement-Lacroix P: Anti-rheumatic activities of histone deacetylase (HDAC) inhibitors in vivo in collageninduced arthritis in rodents. Br J Pharmacol 2007, 150:862-872

14. Blanchard F, Chipoy C: Histone deacetylase inhibitors: new drugs for the treatment of inflammatory diseases? Drug Discov Today 2005, 10: 197-204

15. Vojinovic J, Damjanov N, D’Urzo C, Furlan A, Susic G, Pasic S, Iagaru N, Stefan M, Dinarello CA: Safety and efficacy of an oral histone deacetylase inhibitor in systemic-onset juvenile idiopathic arthritis. Arthritis Rheum 2011, 63:1452-1458

16. Marks PA, Breslow R: Dimethyl sulfoxide to vorinostat: development of this histone deacetylase inhibitor as an anticancer drug. Nat Biotechnol 2007, 25:84-90

17. Chabane N, Zayed N, Afif H, Mfuna-Endam L, Benderdour M, Boileau C, Martel-Pelletier J, Pelletier JP, Duval N, Fahmi H: Histone 
deacetylase inhibitors suppress interleukin-1beta-induced nitric oxide and prostaglandin E2 production in human chondrocytes. Osteoarthritis Cartilage 2008, 16:1267-1274

18. Chen WP, Bao JP, Hu PF, Feng J, Wu LD: Alleviation of osteoarthritis by Trichostatin A, a histone deacetylase inhibitor, in experimental osteoarthritis. Mol Biol Rep 2010, 37:3967-3972

19. Saito T, Nishida K, Furumatsu T, Yoshida A, Ozawa M, Ozaki T: Histone deacetylase inhibitors suppress mechanical stress-induced expression of RUNX-2 and ADAMTS-5 through the inhibition of the MAPK signaling pathway in cultured human chondrocytes. Osteoarthritis Cartilage 2013, 21:165-174

20. Zhong HM, Ding QH, Chen WP, Luo RB: Vorinostat, a HDAC inhibitor, showed anti-osteoarthritic activities through inhibition of iNOS and MMP expression, p38 and ERK phosphorylation and blocking NF-kappaB nuclear translocation. Int Immunopharmacol 2013, 17: 329-335

21. Culley KL, Hui W, Barter MJ, Davidson RK, Swingler TE, Destrument AP, Scott JL, Donell ST, Fenwick S, Rowan AD, Young DA, Clark IM: Class I histone deacetylase inhibition modulates metalloproteinase expression and blocks cytokine-induced cartilage degradation. Arthritis Rheum 2013, 65:1822-1830

22. Venturelli S, Berger A, Bocker A, Busch C, Weiland T, Noor S, Leischner C, Schleicher S, Mayer M, Weiss TS, Bischoff SC, Lauer UM, Bitzer M: Resveratrol as a pan-HDAC inhibitor alters the acetylation status of histone [corrected] proteins in human-derived hepatoblastoma cells. PLoS One 2013, 8:e73097 